\title{
3D Printed Pressure Sensor Based on Surface Acoustic Wave Resonator
}

\author{
Baofa $\mathrm{Hu}^{1,3}$, Zhiwei $\mathrm{Li}^{1,3}$, Yuanjie Wan ${ }^{2,3}$, Peng Zhou ${ }^{1,3}$, Chunquan Zhang ${ }^{1}$, Haisheng San ${ }^{1,2,3, *}$ \\ ${ }^{1}$ Pen-Tung Sah Institute of Micro-Nano Science and Technology, Xiamen University, Xiamen 361005, China, ${ }^{*}$ \\ Corresponding Author / E-mail: sanhs@xmu.edu.cn \\ ${ }^{2}$ School of Electronic Science and Engineering, Xiamen University, Xiamen 361005, China \\ ${ }^{3}$ Shenzhen Research Institute of Xiamen University, Shenzhen 518000, China
}

\begin{abstract}
This paper reports a 3-dimentional (3D) pressure sensor based on surface acoustic wave (SAW) resonators. The SAW resonators were designed and fabricated on $128^{\circ} \mathrm{Y}-\mathrm{X} \mathrm{LiNbO}_{3}$ substrate using the MEMS technology. The pressure sensing structure was 3D-printed using polyactic acid plastic, and two SAW resonators were integrated in the 3D-printed chamber structure for both temperature and pressure sensing. The SAW-based gas pressure sensors demonstrate a sensitivity of $589 \mathrm{ppm} / \mathrm{MPa}$ at the pressure range of $100-600 \mathrm{kPa}$ and temperature of $40^{\circ} \mathrm{C}$.
\end{abstract}

Keywords: Surface acoustic wave, 3D printing, gas pressure sensor; $\mathrm{LiNbO}_{3}$.

\section{INTRODUCTION}

Gas pressure sensors are widely required in the development, production and sale of gas detection alarms, safety and environmental protection testing equipment, industrial production control systems, aviation and navigation equipment, etc. Generally, the sensing mechanisms of gas pressure sensors for commercial applications are based on piezoelectric, piezoresistive, or piezocapacitive effects, etc. [1]-[7]. The signal outputs are current or voltage generally, which responds to the variation of external gas pressure by a change of force, resistance, or capacitance in sensing elements. These sensors should be systematically calibrated and checked for accurate signal outputs. However, shortcomings such as no signal output for the piezoelectricbased gas pressure sensors under the condition of constant pressure, low sensitivity and resolution for the piezoresistivebased gas pressure sensors, and high output impedance for piezocapacitive-based gas pressure sensors are present. These shortcomings would limit their application requirements in high accuracy and sensitive operation.

Surface acoustic wave (SAW) gas pressure sensors are a good candidate for the gas pressure sensing application with the requirements of high accuracy and sensitivity [8]-[9]. The SAW sensors have the properties of small size, high accuracy and sensitivity, radiation hard and low cost, which can be operated with variable frequency and bandwidth in passive wireless mode [10]. SAW is a kind of wave that propagates on the surface of elastic solids. It can be generated by interdigital transducers (IDTs), which are periodic metallic bars deposited on a piezoelectric material. Lord Rayleigh first described and explained the surface mode of acoustic wave propagation in a piezoelectric material [11]-[13]. For Rayleigh type SAWs, the majority of SAW energy is concentrated in the surface region of substrate and in a penetration depth less than one or two wavelengths. Therefore, the SAW propagation characteristics are quite sensitive to the turbulence from external environment. For the SAW-based gas pressure sensors, the change in propagation velocity of SAW will lead to the change of resonance frequency when pressure is exerted on the sensing membrane [14].

Generally, a sealed pressure chamber with sensing elements on the membrane is required in gas pressure sensors. However, it is difficult to fabricate a sealed pressure chamber with sensing piezoelectric membrane due to the incompatibility of piezoelectric materials with the Si-MEMS technique [15]. Thus, there is a need to find a cost-effective approach to fabricate the SAW-based gas pressure sensors. Three-dimensional (3D) printing is a promising technology, which can be used to fabricate a specially designed pressure chamber to integrate the SAW devices [1]. By utilizing an additive process, a 3D structure can be print-fabricated by laying down successive layers of bondable materials, e.g., powdered metal or plastic. Small batch of specific structures 
can be print-fabricated with lower cost and faster time. Combining 3D printing pressure sensitive structure and SAW resonators, the pressure sensors can be tailored for specific purposes.

In this paper, a SAW resonator was designed and fabricated on $128^{\circ} \mathrm{Y}-\mathrm{X} \mathrm{LiNbO}_{3}$ substrate using the MEMS technology. The structure of the pressure sensing chamber was designed using the finite element method for simulating the dependence of resonance frequency on pressure. The pressure chamber structure was 3D-printed using polyactic acid plastic, and two SAW resonators were integrated in the $3 \mathrm{D}$ printed chamber structure for both temperature and pressure sensing. The SAW-based gas pressure sensors demonstrate high sensitivity at wide pressure range of $100-600 \mathrm{kPa}$.

\section{DESIGN \& EXPERIMENT}

Lithium niobate $\left(\mathrm{LiNbO}_{3}\right)$ is a single crystal piezoelectric material that has been widely used in SAW devices due to its higher electromechanical coupling coefficient, low attenuation and moderate temperature coefficient. In this work, the $128^{\circ} \mathrm{Y}-\mathrm{X} \mathrm{LiNbO}_{3}$ was used as the piezoelectric substrate, and the single-port SAW resonator was fabricated on the substrate. Fig.1.a) shows the schematic structure of the designed single-port SAW resonator. An IDT consists of a series of interleaved electrodes made of a metal film deposited on a piezoelectric substrate, and a pair of grating reflectors are arranged on both sides of the IDT [16]. The excited resonant wavelength $\lambda$ is determined by IDT period spacing $p_{i}$ and acoustic velocity $v$. The resonant frequency $f$ of the SAW resonator can be expressed as:

$$
f=\frac{v}{\lambda}=\frac{v}{p_{i}}
$$

As pressure is exerted on the back surface of the SAW resonator, a convex deformation is generated in the piezoelectric substrate, thereby leading to a tensile strain at the surface of the piezoelectric substrate with IDT electrodes. The deformation causes two effects on resonance frequency of the SAW resonator. Firstly, nonlinear deformation due to the pressure overload will affect the stiffness coefficient and elastic modulus of the piezoelectric material, thereby resulting in the variation of wave velocity of the SAW propagation [17]. Secondly, the deformation leads to the variation of width between IDT fingers, which further affect the period of the IDT, as shown in Fig.1.b). The combination of the above two influence factors enables the variation of resonant frequency of the SAW resonator, which can be expressed by the following differential equation [18]:

$$
\mathrm{d} f=\frac{\mathrm{d} v}{p_{i}}-\frac{v}{p_{i}^{2}} \mathrm{~d} p_{i}
$$

For a linear deformation, the strain has little effect on the phase velocity of the SAW, and thus the phase velocity $\mathrm{d} v$ can be neglected, so the frequency variation can be simplified as

$$
\mathrm{d} f \approx-\frac{v}{p_{i}^{2}} \mathrm{~d} p_{i}
$$

where $\mathrm{d} p_{i} / p_{i}$ represents strain $S$, so the formula (3) can be expressed as

$$
\mathrm{d} f \approx-f S
$$

It can be seen from equation (4) that the frequency offset is equal to $S$.
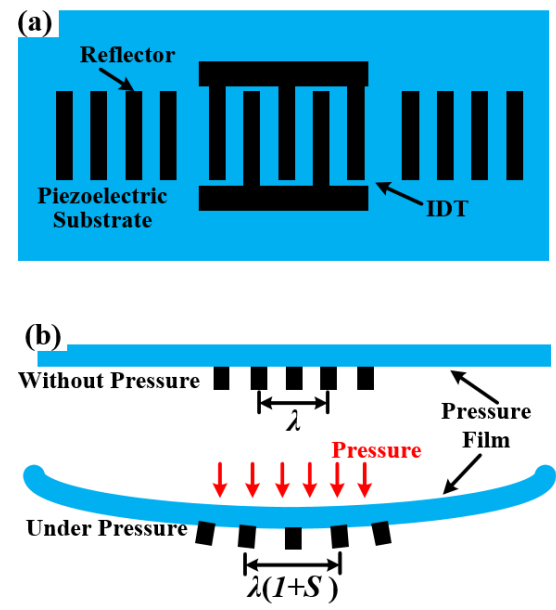

Fig.1. a) Schematic structure of the single-port SAW resonator; b) the pressure-generated convex deformation and its influence on the period of the IDT

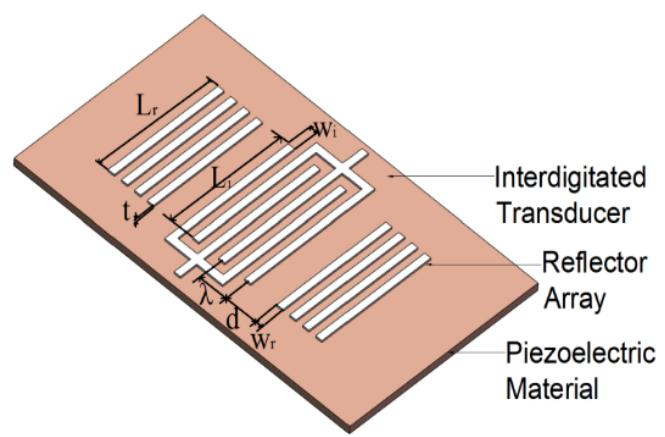

Fig.2. Schematic structure and size parameter of SAW resonator.

Fig.2. shows the schematic structure and size parameter of the SAW resonator. The design parameters of SAW resonator based on $128^{\circ} \mathrm{Y}-\mathrm{X} \mathrm{LiNbO}_{3}$ substrates are presented in Table 1., which were optimized by finite element simulation and COM (coupling-of-modes) calculation [19], [20]. The width of IDT and the grating reflector were determined to be $2 \mu \mathrm{m}$, corresponding to an IDT period of $8 \mu \mathrm{m}$ for a resonation frequency around $500 \mathrm{MHz}$. Based on the tradeoff consideration of device size and quality factor $\mathrm{Q}$, there are 50 pairs of IDTs and 100 pairs of grating reflectors with an IDT aperture of $80 \lambda$ [21]. For good conductivity and low mass loading, a $100 \mathrm{~nm}$ thick Al was designed to fabricate IDTs and reflectors [22]. The distance between IDT and reflector was adjusted to $16 \mu \mathrm{m}$ so that the peak of IDT conductance lies in the center of the stop-band of reflectors [20]. 
Table 1. Design parameters of SAW resonator.

\begin{tabular}{|c|c|c|}
\hline Parameter & Symbol & value \\
\hline Bar-width of IDT & $W_{i}$ & $2 \mu \mathrm{m}$ \\
\hline Bar-width of reflector & $W_{r}$ & $2 \mu \mathrm{m}$ \\
\hline Period of IDT & $P_{i}$ & $8 \mu \mathrm{m}$ \\
\hline $\begin{array}{c}\text { Length of IDT } \\
\text { aperture }\end{array}$ & $L_{i}$ & $800 \mu \mathrm{m}$ \\
\hline $\begin{array}{c}\text { Length of reflector } \\
\text { Pairs of IDT }\end{array}$ & $L_{r}$ & $800 \mu \mathrm{m}$ \\
\hline $\begin{array}{c}\text { Bar number of } \\
\text { reflector }\end{array}$ & $N_{r}$ & 100 \\
\hline $\begin{array}{c}\text { Distance between IDT } \\
\text { and reflector }\end{array}$ & $d$ & $16 \mu \mathrm{m}$ \\
\hline $\begin{array}{c}\text { Metallization of } \\
\text { IDT/reflector }\end{array}$ & $\eta$ & $50 \%$ \\
\hline $\begin{array}{c}\text { Thickness of } \\
\text { electrode/reflector }\end{array}$ & $t$ & $100 \mathrm{~nm}$ \\
\hline
\end{tabular}

Generally, the center frequency of the SAW device will vary with the change of temperature, and the extent of frequency offset will be affected by the temperature coefficient of piezoelectric materials [23]. Therefore, it is necessary to compensate the temperature-induced frequency offset by a differential system, in which two matched SAWbased sensors are used back-to-back to provide compensation for ambient temperature changes. One SAW-based sensor is sensitive to the pressure and temperature and the other is a reference sensor only sensitive to the temperature. By a subtraction for the two frequency shift, the temperature effect can be cancelled out. Therefore, two chambers are required when designing the package structure, one chamber is the pressure chamber and the other is the reference chamber for temperature calibration. Two chambers have the same cavity size but different membrane thickness. Two SAW resonators are arranged in the center position of the membrane and are sealed in two different cavities, respectively. For the pressure sensing chamber, the membrane thickness is very thin so that a tensile strain is generated due to external gas pressure on the membrane, resulting in the frequency shift of the SAW resonator. By contrast, the thickness of membrane in the reference chamber is so large that the external pressure does not induce significant strain in the membrane and thus the frequency shift.

The sensing package structure comprises two parts, namely membrane part and cavity part, as shown in Fig.3., the upper membrane part consists of two different thicknesses of membrane structures, and lower cavity part consists of two identical chambers fabricated in a substrate. As shown in Fig.3.a), a 3D printer (JDM-3DP200) was used to fabricate the two parts using the fused deposition modeling (FDM) technique. Finally, the two parts were adjoined together using glue. The JDM-3DP200 printer is suitable for printing polyactic acid plastic, which is a thermoplastic aliphatic polyester organic material with good mechanical and processing properties. More importantly, the polyactic acid plastic is an environmentally friendly and commercially available material, which can be rapidly degraded by hydrolysis, pyrolysis or natural degradation. For a good adhesion between the SAW resonator and a polyactic acid plastic membrane, ethyl cyanoacrylate (ECA) is used as adhesive material due to its high cohesive strength and low viscosity.

The size of SAW resonator is $3 \mathrm{~mm} \times 2 \mathrm{~mm} \times 0.5 \mathrm{~mm}$ (length $\times$ width $\times$ depth), and the cavity size is designed to be $6 \mathrm{~mm} \times 4 \mathrm{~mm} \times 2 \mathrm{~mm}$, and the thickness of the side wall of the chambers is set to $2 \mathrm{~mm}$, as shown in Fig.3.b). The size of the membrane part is $21 \mathrm{~mm} \times 10 \mathrm{~mm} \times 0.5 \mathrm{~mm}$ (length $\times$ width $\times$ height), and the thicknesses of the pressure sensing membrane and the reference membrane are $0.5 \mathrm{~mm}$ and $1.5 \mathrm{~mm}$, respectively.
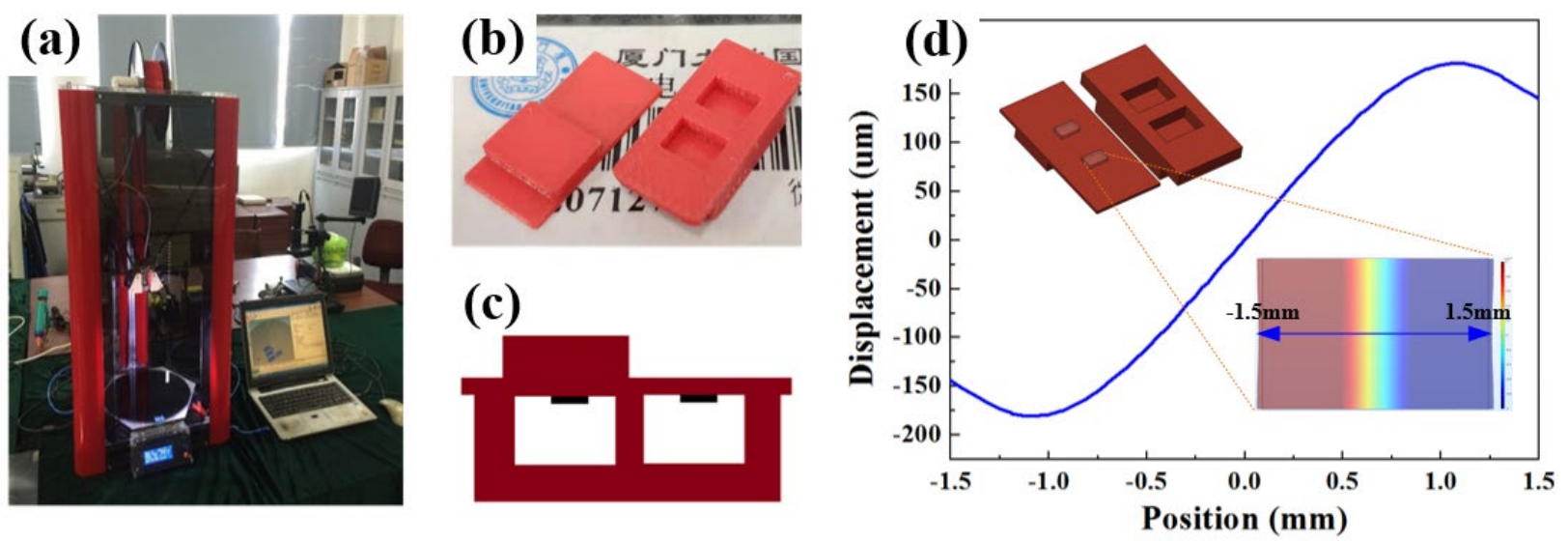

Fig.3. 3D printer (JDM-3DP200) a); sample of 3D-printed structure parts b); schematic of package structure c); deformation map of $\mathrm{LiNbO}_{3}$ substrate and its dependence of deformation on position d). 

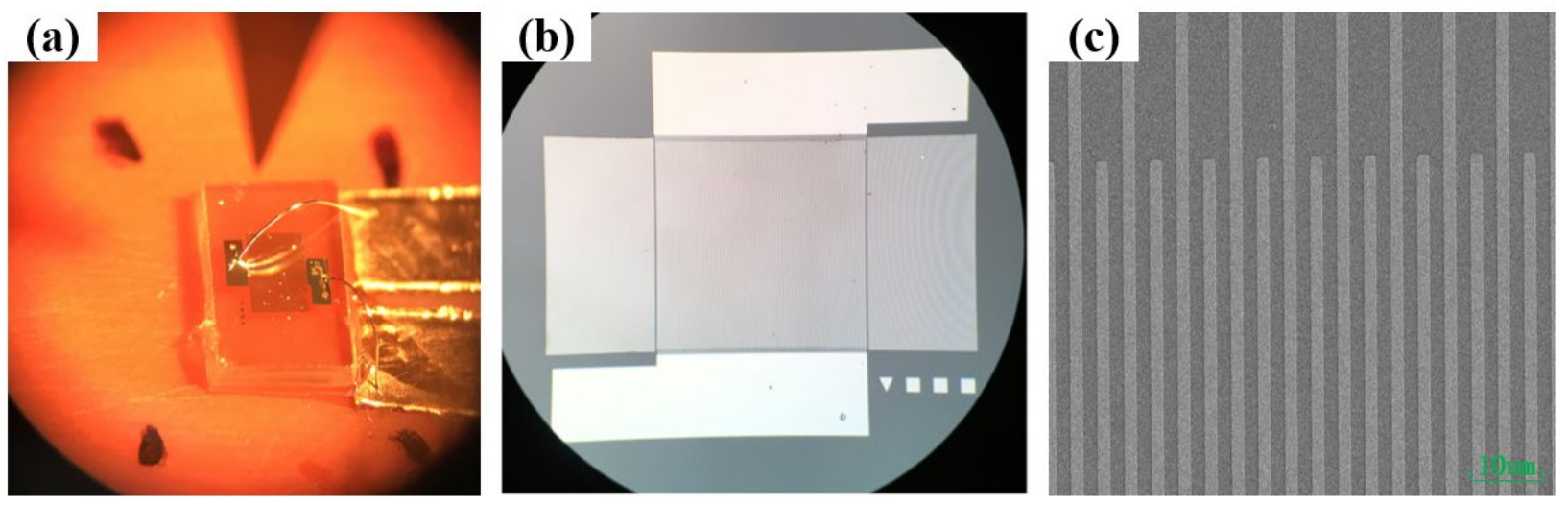

Fig.4. Electrical connection between the SAW resonator and the microstrip line using wire bonding a); microstructure images of SAW resonator and IDT by optical microscope b) and scanning electron microscope c).
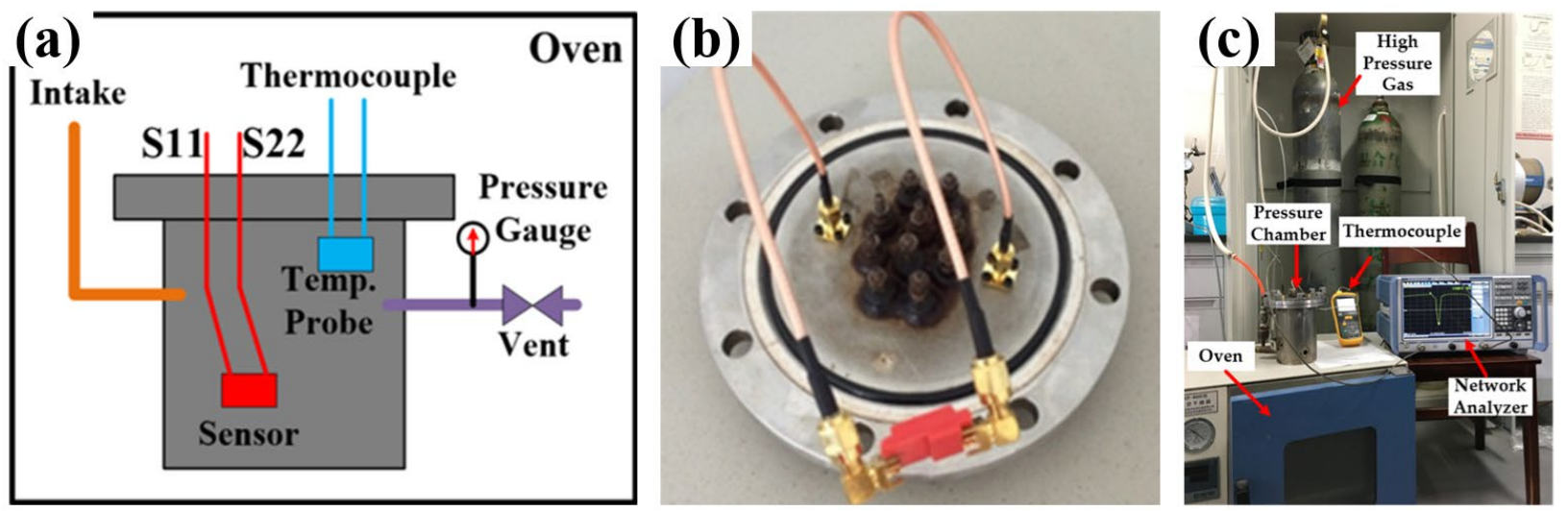

Fig.5. Schematic diagram of PMV a); SAW-based pressure sensor connected to the cover of PMV through the coaxial lines and SMA connectors $b$ ), pressure measuring system $c$ ).

To gain insight into the effect of gas pressure on the SAW resonator attached to the polyactic acid plastic film, COMSOL software is used to analyze the deformation of piezoelectric substrate of the SAW resonator when a gas exerts pressure on the polyactic acid plastic film. It is assumed that the SAW resonators are rigidly attached to the polyactic acid plastic film. Fig.3.d) shows the tensile deformation map on the surface of the $\mathrm{LiNbO}_{3}$ substrate with IDT, and the dependence of deformation displacement on position. From the simulation results of the deformation map, the strain can be calculated and the frequency shift can be further estimated. According to the design parameters of the pressure chamber structure, the strain on the $\mathrm{LiNbO}_{3}$ substrate surface is approximately $0.5725 \times 10^{-4}$ per $100 \mathrm{kPa}$. According to equation (4) and the simulation results, the frequency sensitivity of the SAW pressure sensor can be estimated as $572.5 \mathrm{ppm} / \mathrm{MPa}$.

In order to build an electrical connection between the SAW resonator sealed in chamber and external small A type (SMA) connector, it is necessary to use a metal film material to form microstrip waveguide lines. In this work, an adhesive-backed copper foil was used to form the microstrip lines due to its low cost and high conductivity. The SAW resonator and the microstrip line were connected using wire bonding technique, as shown in Fig.4.a). The microstrip lines were led out of the interior of the chamber, and the buried microstrip lines in adhesive region did not result in any damage of the sealing structure. Fig.4.b) and Fig.4.c) show the microstructure images of SAW resonator and IDT by optical microscope and scanning electron microscope, respectively. Finally, the SAW-based pressure sensor was completed by structure adhesive packaging in nitrogen atmosphere, and the SMA connectors were soldered to the exposed microstrip lines.

Fig.5.a) shows the schematic diagram of a pressure measuring vessel (PMV). Two radio frequency SMA adapters are fixed in the upper cover of the PMV. An air intake and an air vent are arranged on two side of the PMV, and a high-precision pressure gauge is fixed in the vent pipe for detecting the real-time pressure value. To measure the gas pressure under different temperatures, the PMV is placed in an oven to provide a controlled temperature environment. A thermocouple probe is placed in the PMV to calibrate the temperature. Fig.5.b) shows the packaged SAW-based pressure sensor connected to the SMA connectors in the cover of the PMV through the coaxial lines. Fig.5.c) displays the photo of the measuring system. The sensing signals are measured by a network analyzer. A $20 \mathrm{MPa}$ high-pressure nitrogen gas cylinder is used as a pressure source, and a pressure reducing valve is used to control the maximum of gas pressure for the PMV. 


\section{RESULTS \& DISCUSSION}

For a single-port SAW resonator, the $\mathrm{S}_{11}$ signal is used to characterize the device performance. Generally, the maximum energy conversion from electrical energy to mechanical energy occurs at the resonant frequency point of the SAW resonators. Once working frequency deviated from the resonant frequency of the device, the electrical signal cannot be converted into the mechanical energy of the piezoelectric material due to the impedance mismatch, resulting in a small signal output of return loss. Fig.6.a) shows the resonance peaks of the SAW resonators in pressure sensor chamber and reference chamber, respectively. It can be seen that two SAW resonators have the same resonance frequency of about $495 \mathrm{MHz}$.
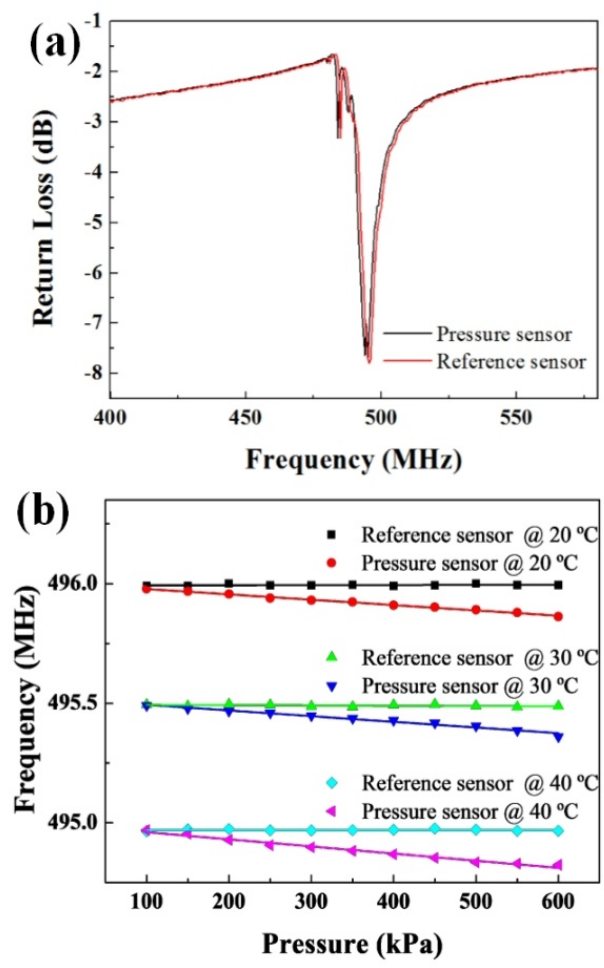

Fig.6. Return loss of pressure and reference SAW resonator sensors at $100 \mathrm{kPa}$ a) and the response of the frequency vs. pressure at different temperatures $b$ ).

The SAW-based pressure sensors were measured with temperature range from $20{ }^{\circ} \mathrm{C}$ to $40{ }^{\circ} \mathrm{C}$, and pressure range from 100 to $600 \mathrm{kPa}$, as shown in Fig.6.b). When temperature increases from $20{ }^{\circ} \mathrm{C}$ to $40{ }^{\circ} \mathrm{C}$, the resonant frequency decreases from $495.994 \mathrm{MHz}$ to $494.970 \mathrm{MHz}$, the temperature coefficient is $103.434 \mathrm{ppm} /{ }^{\circ} \mathrm{C}$. At $20{ }^{\circ} \mathrm{C}, 30^{\circ} \mathrm{C}$, and $40^{\circ} \mathrm{C}$, the frequency shifts of sensor under $500 \mathrm{kPa}$ pressure change are $-0.1158 \mathrm{MHz},-0.1308 \mathrm{MHz}$, and $0.1458 \mathrm{MHz}$, respectively. The sensitivities of pressure sensor are $467 \mathrm{ppm} / \mathrm{MPa}, 528 \mathrm{ppm} / \mathrm{MPa}$, and $589 \mathrm{ppm} / \mathrm{MPa}$, respectively. These results are in line with the sensitivity calculated by the previous simulation. Experimental results show that the resonant frequency decreases with the increase of temperature or pressure, which should be attributed to both temperature and stress dependent SAW properties.
Furthermore, the reference SAW-based sensor has no frequency response when the external gas pressure varies, which means the pressure sensor can be calibrated by temperature compensation of reference sensor. In order to achieve a wider temperature range of application, 3D printing materials with high temperature resistance can be used, such as acrylonitrile butadiene styrene (ABS), ceramics, metals, etc. Meanwhile, 3D printing equipment with better performance is also required [24].

\section{CONCLUSION}

In this paper, we report an integrated pressure sensor based on 3D printed chamber and two highly sensitive SAW resonators. The structure of the pressure sensing chamber was designed using the finite element method for simulating the dependence of resonance frequency on pressure, and the $3 \mathrm{D}$ printing technique was used to fabricate the pressure sensing structure using polyactic acid plastic. The SAW resonators were designed and fabricated on $128^{\circ} \mathrm{Y}-\mathrm{X} \mathrm{LiNbO}_{3}$ substrate using the MEMS technology, and two SAW resonators were integrated in the 3D-printed chamber structure for both temperature and pressure sensing. The frequency shift of $20{ }^{\circ} \mathrm{C}$ to $40{ }^{\circ} \mathrm{C}$ is $-1.024 \mathrm{MHz}$, so the temperature coefficient is $103.434 \mathrm{ppm} /{ }^{\circ} \mathrm{C}$. The maximum frequency shift for the full pressure range of $500 \mathrm{kPa}$ is $-0.1458 \mathrm{MHz}$ at $40{ }^{\circ} \mathrm{C}$, and the sensitivity is $589 \mathrm{ppm} / \mathrm{MPa}$. It is expected that the $3 \mathrm{D}$ printed pressure sensors can be used for specific purposes with some special requirements in pressure range, low-cost, and fast fabrication.

\section{ACKNOWLEDGMENT}

This work was supported by the following programs: the National Natural Science Foundation of China (Grant No. 61574117) and the Natural Science Foundation of Guangdong Province (Grant No. 2018B030311002).

\section{REFERENCES}

[1] Faller, L.M., Granig, W., Krivec, M., Abram, A., Zangl, H. (2018). Rapid prototyping of force/pressure sensors using 3D- and inkjet-printing. Journal of Micromechanics and Microengineering, 28, 104002.

[2] Davidovikj, D., Scheepers, P.H., van der Zant, H.S.J., Steeneken, P.G. (2017). Static capacitive pressure sensing using a single graphene drum. ACS Applied Materials \& Interfaces, 9 (49), 43205-43210.

[3] San, H., Song, Z., Wang, X., Zhao, Y. (2012). Piezoresistive pressure sensors for harsh environments Optics and Precision Engineering, 20 (3), 550-555.

[4] Gajula, D.R., Jahangir, I., Koley, G. (2018). High temperature $\mathrm{AlGaN} / \mathrm{GaN}$ membrane based pressure sensors. Micromachines, 9 (5), 207.

[5] Shtyrkov, O.V., Yushkov, V.A. (2016). A dual absolute pressure measuring transducer. Instruments and Experimental Techniques, 59, 139-141.

[6] Murawski, K. (2015). New vision sensor to measure gas pressure. Measurement Science Review, 15 (3), 132138. 
[7] Hu, B., Li, Z., Wan, Y., Li, M., San, H. (2021). Gas pressure measurement using micro-corona-discharging effect in surface acoustic wave resonators. Results in Physics, 25, 104221.

[8] Nicolay, P., Elmazria, O., Sarry, F., Bouvot, L., Marche, N., Kambara, H. (2008). Innovative surface acoustic wave sensor for accurate measurement of subatmospheric pressure. Applied Physics Letters, 92, 141909.

[9] Oh, H., Lee, K., Eun, K., Choa, S., Yang, S. (2012). Development of a high-sensitivity strain measurement system based on a SH SAW sensor. Journal of Micromechanics and Microengeering, 22, 025002.

[10] Dai, X., Fang, L., Zhang, C., Sun, H. (2020). An impedance-loaded orthogonal frequency-coded SAW sensor for passive wireless sensor networks. Sensors, 20 (7), 1876.

[11] Royer, D., Dieulesaint, E. (2000). Elastic Waves in Solids II: Generation, Acousto-optic Interaction, Applications. Spinger, 336-342. ISBN 9783540659310.

[12] Hu, B., Zhang, S., Zhang, H., Lv, W., Zhang, C., Lv, X., San, H. (2017). Fabrications of $L$-Band LiNbO3based SAW resonators for aerospace applications. Micromachines, 10 (6), 349.

[13] Zhou, P., Chen, C., Wang, X., Hu, B., San, H. (2018). 2-Dimentional photoconductive MoS2 nanosheets using in surface acoustic wave resonators for ultraviolet light sensing. Sensors and Actuators A: Physical, 271 (1), 389-397.

[14] Xie, L., Wang, T., Xing, J., Zhu, X. (2018). An embedded surface acoustic wave pressure sensor for monitoring civil engineering structures. IEEE Sensors Journal, 18 (13), 5232-5237.

[15] Dixon, B., Kalinin, V., Beckley, J., Lohr, R. (2006). A second generation in-car tire pressure monitoring system based on wireless passive SAW sensors. In IEEE International Frequency Control Symposium. IEEE.
[16] Tanski, W.J. (1978). A configuration and circuit analysis for one-port SAW resonators. Journal of Applied Physics, 49 (4), 2559.

[17] Jasek, K., Pasternak, M. (2015). The influence of external pressure on resonant frequency of SAW resonator. Acta Physica Polonica A, 127 (6), 16011605.

[18] Hara, B., Mitsui, M., Sano, K., Nagasawa, S., Kuwano, H. (2012). Experimental study of highly sensitive sensor using a surface acoustic wave resonator for wireless strain detection. Japanese Journal of Applied Physics, 51, $07 \mathrm{GC} 23$.

[19] Hofer, M., Finger, N., Kovacs, G., Schoberl, J., Langer, U., Lerch, R. (2002). Finite element simulation of bulkand surface acoustic wave (SAW) interaction in SAW devices. In IEEE Symposium (IUS) Ultrasonics. IEEE, 53-56.

[20] Kannan, T. (2006). Finite element analysis of surface acoustic wave resonators. M.S. Thesis, Department of Electrical Engineering, University of Saskatchewan, Saskatoon, Saskatchewan, Canada.

[21] Hashimoto, K.-Y. (2000). Surface Acoustic Wave Devices in Telecommunications: Modelling and Simulation. Springer, ISBN 978-3-540-67232-6.

[22] Haydl, W.H., Hiesinger, P., Smith, R.S., Dischler, B., Heber, K. (1976). Design of quartz and lithium niobate SAW resonators using aluminum metallization. In 30th Annual Symposium on Frequency Control. IEEE.

[23] Hoummady, M., Hauden, D. (1994). Acoustic wave thermal sensitivity: Temperature sensors and temperature compensation in microsensors. Sensors and Actuators A: Physical, 44 (3), 177-182.

[24] Pandian, A., Belavek, C. (2016). A review of recent trends and challenges in 3D printing. In Proceedings of the 2016 ASEE North Central Section Conference. American Society for Engineering Education.

Received March 30, 2021 Accepted May 31, 2021 